\title{
A Trivariate Extreme Value Distribution Applied to Flood Frequency Analysis
}

\section{Carlos A. Escalante-Sandoval}

Engineering Graduate Studies Division, Universidad Nacional Autonoma de Mexico, 04510 Mexico, DF, Mexico

and

Jose A. Raynal-Villasenor Institute of Sciences, Universidad Autonoma de Chihuahua, 31800 Chihuahua, Chih., Mexico
A trivariate extreme value distribution has been derived from the logistic model for the multivariate extreme value distribution. The construction of its corresponding probability distribution and density function is described. In order to obtain the parameters of such a trivariate distribution, a generalized maximum likelihood estimation procedure is described to allow for lite cases of samples with different record lengths. Furthermore the rcliability of the estimated parameters of the Irivariate extreme value distribulion is measured lhrough the use of relative information ratios. A region in Northern Mexico with six gauging slations has been selected to apply the trivariate model. Results produced by the proposed model have been compared with those oblained by general extreme value (GEV) distribution functions,

Key words: Jistribution functions; distribulion models; fhood frequency analysis; method of maximum likelihood; trivariate cxtreme value distributions.

Accepted: March 22, 1994

\section{Introduction}

Flood frequency analysis has been carried out by using univariate distribution functions, the extreme value distributions being an important set of distributions used in this field of study. Generally, parameters of such distributions are estimated from a short record of flows. The variability of these estimates has prompted exploration of joint estimation models which use information from streamflow records of neighboring gauging stations.

In pioneering papers Finkelstein [1], Tiago de Oliveira [2], and Gumbel [3] gave the foundations for the multivariate approach to extreme value distributions. Following this work, several bivatiate extreme value models began to appear in the literature. Rueda [4] explored the logistic and mixed models for bivariate extreme value distributions when both marginals are extreme value type I (EVI) distributions. He reported improvements in the estimation of parameters when the bivariate approach is used. Raynal [5] developed and applied three bivariate options from the logistic model of bivariate extreme value distribution for flood frequency analysis. He found that there exists an improvement in the parameter estimation phase, even in the case when both samples have the same record lengths.

Herein, the trivariate approach of multivariate extreme value distribution is presented with a view to its application to flood frequency analysis.

General characteristics, the procedure for estimation, and reliability of parameters of the trivariate extreme value distributions will be described in the following sections. An actual application of the proposed model to six gauging stations in Northern Mexico is presented in the paper. 


\section{Characteristics of the Trivariate Logis- tic Model}

From the multivariate extension of the logistic model for bivariate extreme value distribution [3], the trivariate approach is:

$$
\begin{gathered}
F(x, y, z, \underline{\theta})=\exp \left\{-\left[(\ln F(x))^{m}\right.\right. \\
\left.+(-\ln F(y))^{m+}+(-\ln F(z))^{m}\right\}^{1 / m},
\end{gathered}
$$

where $m$ is the association parameter $(m \geq 1)$ and $F(s)=F(s, \underline{\theta})$ is the marginal distribution function of $s$. Equation (1) must satisfy the following inequalities (Tiago de Oliveira $[6,7]$ ):

$F(x) F(y) F(z) \leq F(x, y, z) \leq \min [F(x), F(y), F(z)]$

$$
\begin{gathered}
{[F(x, y,) F(x, z) F(y, z)]^{\frac{1}{4}}} \\
\leq F(x, y, z) \leq \frac{[F(x, y) F(x, z) F(y, z)]^{1 / 2}}{[F(x) F(y) F(z)]^{1}} .
\end{gathered}
$$

Marginals in Eq. (1) can be either EVI distributions:

$$
F(s)=\exp \left(-\exp -\left(\frac{s-u}{\alpha}\right)\right)
$$

or GEV distributions:

$$
F(s)=\exp -\left(1-\left(\frac{s-u}{\alpha}\right) \beta\right)^{1 / \beta}
$$

The combinations have been named (Escalante [8]):

a) Trivariate extreme value distribution type 111 (TEV111) or TriGumbel distribution. All marginals are EVI distributions.

b) Trivariate extreme value distribution type 112 (TEV112) or BiGumbel-GEV distribution.

c) Trivariate extreme value distribution type 122 (TEV122) or BiGEV-EVI distribution.

d) Trivariate extreme value distribution type 222 (TEV222) or TriGumbel distribution. All marginals are GEV distributions.

The particular form of Eq. (1), when the marginals are GEV distributions for the maxima, is (Escalante [8]):

$F\left(x, y, z, u_{1}, \alpha_{1}, \beta_{1}, u_{2}, \alpha_{2}, \beta_{2}, u_{1}, \alpha_{3}, \beta_{3}, m_{\mathrm{f}}\right)=$

$$
\begin{gathered}
\exp \left[-\left(\left(1-\left(\frac{x-u_{1}}{\alpha_{1}}\right) \beta_{1}\right)^{m_{3} / \beta_{1}}\right.\right. \\
+\left(1-\left(\frac{y-u_{2}}{\alpha_{2}}\right) \beta_{2}\right)^{m m_{1} / \beta_{3}} \\
\left.\left.+\left(1-\left(\frac{z-u_{3}}{\alpha_{3}}\right) \beta_{3}\right) m^{m_{2} / \beta_{3}}\right)^{1 / / m_{1}}\right]
\end{gathered}
$$

where $u_{i}, \alpha_{i}$ and $\beta_{i}, i=1,2,3$, are the location, scale and shape parameters of the marginal GEV distributions for the maxima. The corresponding probability density function is (Escalante [8]):

$$
\begin{aligned}
& f\left(x, y, z, u_{1}, \alpha_{1}, \beta_{1}, u_{2}, \alpha_{2}, \beta_{3}, u_{3}, \alpha_{3}, \beta_{3}, m_{1}\right)=\frac{1}{\alpha_{1} \alpha_{2} \alpha_{3}}\left(1-\left(\frac{x-u_{1}}{\alpha_{1}}\right) \beta_{1}\right)^{m_{1} / \beta_{1}-1}\left(1-\left(\frac{y-u_{2}}{\alpha_{2}}\right) \beta_{2}\right)^{m_{1} / \beta_{2}-1} \\
& \left(1-\left(\frac{z-u_{3}}{\alpha_{3}}\right) \beta_{3}\right)^{m_{t} / \beta_{3}-1} \exp \left[-\left(\left(1-\left(\frac{x-u_{1}}{\alpha_{1}}\right) \beta_{1}\right)^{m m_{4} / \beta_{1}}+\left(1-\left(\frac{y-u_{2}}{\alpha_{2}}\right) \beta_{2}\right)^{m_{5} / \beta_{2}}+\left(1-\left(\frac{z-u_{3}}{\alpha_{3}}\right) \beta_{3}\right)^{m_{1} / \beta_{3}}\right)^{1 / m_{1}}\right]
\end{aligned}
$$

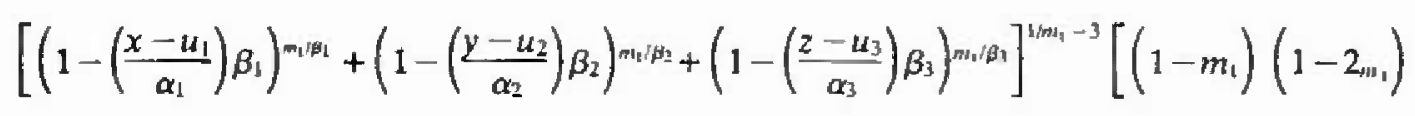

$$
\begin{aligned}
& +\left(\left(1-\left(\frac{x-u_{1}}{\alpha_{1}}\right) \beta_{1}\right)^{n_{4} / \beta_{1}}+\left(1-\left(\frac{y-u_{2}}{\alpha_{2}}\right) \beta_{2}\right)^{m_{1} / \beta_{2}}+\left(1-\left(\frac{z-u_{3}}{\alpha_{3}}\right) \beta_{3}\right)^{m_{1} / \beta_{3}}\right)^{2 / m_{1}}+\left(3 m_{1}-3\right) \\
& \left.\left(\left(1-\left(\frac{x-u_{1}}{\alpha_{1}}\right) \beta_{1}\right)^{m m_{2} / \mu_{3}}+\left(1-\left(\frac{y-u_{2}}{\alpha_{2}}\right) \beta_{2}\right)^{m m_{1} / \beta_{2}}+\left(1-\left(\frac{z-u_{3}}{\alpha_{3}}\right) \beta_{3}\right)^{m m_{1} / \beta_{3}}\right)^{1 / m_{1}}\right] .
\end{aligned}
$$




\section{Journal of Research of the National Institute of Standards and Technology}

\section{Estimation of Parameters}

The method of maximum likelihood for estimating the parameters of trivariate extreme value distributions has been chosen due to its characteristics for consistency in large sample estimation and applicability in estimating the parameters of cumbersome density functions.

For the case of trivariate distribution functions, the sample arrangements could allow having either an equal or different record length in any of the samples to be analysed.

In order to consider all possible combinations of data, it is required to have a sufficiently flexible formulation, therefore the following general form of the likelihood function will be used based on the generalization obtained by Anderson [9]:

$$
\begin{aligned}
& L\left(x, y, z_{1} \underline{\theta}\right)=\left[\prod_{i=1}^{r_{1}} f\left(p_{i}, \underline{\theta}_{1}\right)\right]^{h_{1}}\left[\prod_{i=1}^{\mu_{2}} f\left(p_{i}, q_{i}, \underline{\theta}_{2}\right)\right]^{h_{2}} \\
& {\left[\prod_{i=1}^{n_{3}} f\left(x, y, z, \underline{\theta}_{3}\right)\right]^{b_{5}}\left[\prod_{i=1}^{n_{4}} f\left(r_{i}, s_{i}, \underline{\theta}_{4}\right)\right]^{h_{4}}\left[\prod_{i=1}^{n_{5}} f\left(r_{i}, \underline{\theta}_{5}\right)\right]^{I_{5}},}
\end{aligned}
$$

where:

$$
\begin{aligned}
n_{1}, n_{2}= & \text { are respectively the univariate and } \\
& \text { bivariate record lengths before the } \\
& \text { conmon period } n_{3,} \\
n_{4}, n_{5}= & \text { are respectively the bivariate and uni- } \\
& \text { variate record lengths after the com- } \\
& \text { mon period } n_{3},
\end{aligned}
$$

$p \quad=$ is the variable with univariate record before the common period,

$(p, q)=$ are the variables with bivariate record before the common period,

$(x, y, z)=$ are the variables with trivariate record during the common period,

$(r, s)=$ are the variables with bivariate record after the common period,

$r \quad=$ is the variable with univariate record after the common period,

$I_{i} \quad=$ are indicator numbers such that: $I_{i}=1$ if $n_{i}>0$ and $I_{i}=0$ if $n_{i}=0$.

The logarithmic function will be used instead of the likelihood function. So, Eq. (8) is transformed into:

$$
\begin{gathered}
L L\left(x_{5}, y, z, \underline{\theta}\right)=I_{1}\left[\sum_{i=1}^{n_{1}} \ln f\left(p_{i}, \underline{\theta}_{1}\right)\right] \\
+I_{2}\left[\sum_{i=1}^{n_{2}} \ln f\left(p_{i}, q_{i}, \underline{\theta}_{2}\right)\right]+I_{3}\left[\sum_{i=1}^{m_{3}} \ln f\left(x_{1}, y, z_{i} \underline{\theta}_{3}\right)\right] \\
+I_{4}\left[\sum_{i=1}^{n_{4}} \ln f\left(r_{i}, s_{i}, \underline{\theta}_{4}\right)\right]+I_{5}\left[\sum_{i=1}^{n_{5}} \ln f\left(r_{i}, \underline{\theta}_{5}\right)\right] .
\end{gathered}
$$

The maximum likelihood estimators of parameters for the trivariate extreme value distributions are those values for which Eq. (9) is maximized.

The corresponding logarithmic likelihood function for the trigeneral extreme value (TEV222) distribution function, based on Eq. (9) from [8] is shown in Eq. (10):

$$
\begin{aligned}
& L L\left(x, y, z_{1} u_{1}, \alpha_{1}, \beta_{l}, u_{2}, \alpha_{2}, \beta_{2}, u_{3}, \alpha_{3}, \beta_{3}, m_{1}, m_{b l}, m_{b 2}\right)=I_{1}\left\{-n_{1} \ln \alpha_{p}\right. \\
& \left.+\sum_{i=1}^{n_{1}}\left[-\left(1-\left(\frac{p_{i}-u_{p}}{\alpha_{p}}\right) \beta_{p}\right)^{1 / \beta_{p}}+\ln \left(1-\left(\frac{p_{i}-u_{p}}{\alpha_{p}}\right) \beta_{p}\right)^{1 / \beta_{p}-1}\right]\right\} \\
& +I_{2}\left\{\sum _ { i = 1 } ^ { m _ { 2 } } \left[-\left(\ln \alpha_{y_{1}}+\ln \alpha_{q}\right)+\ln \left(1-\left(\frac{p_{i}-u_{p}}{\alpha_{p}}\right) \beta_{p}\right)^{m_{m_{1} / \beta_{p}}-1}+\ln \left(1-\left(\frac{q_{i}-u_{q}}{\alpha_{q}}\right) \beta_{i}\right)^{m_{m_{1}} / \beta_{q}-1}\right.\right.
\end{aligned}
$$

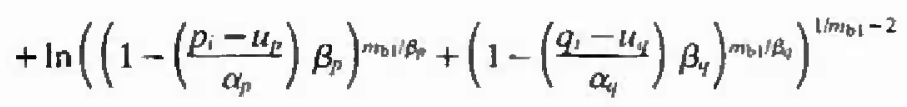

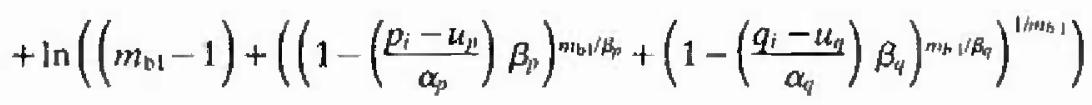

$$
\begin{aligned}
& \left.\left.-\left(\left(1-\left(\frac{p_{i}-u_{p}}{\alpha_{p}}\right) \beta_{p}\right)^{m_{b} / \beta_{p}}+\left(1-\left(\frac{q_{i}-u_{q}}{\alpha_{y}}\right) \beta_{q}\right)^{m+1 / \beta_{p}}\right)^{1 / m_{b-1}}\right]\right\}
\end{aligned}
$$




$$
\begin{aligned}
& +J_{3}\left\{-n_{3}\left(\ln \alpha_{1}+\ln \alpha_{2}+\ln \alpha_{3}\right)+\sum_{i=1}^{n_{3}}\left[\ln \left(1-\left(\frac{x_{i}-u_{1}}{\alpha_{1}}\right) \beta_{1}\right)^{m_{1} / \beta_{1}-1}\right.\right. \\
& +\ln \left(1-\left(\frac{y_{i}-u_{2}}{\alpha_{2}}\right) \beta_{2}\right)^{m_{1} / \beta_{2}-1}+\ln \left(1-\left(\frac{z_{i}-u_{3}}{\alpha_{3}}\right) \beta_{3}\right)^{m / \beta_{3}-1} \\
& +\ln \left(\left(1-\left(\frac{x_{j}-u_{1}}{\alpha_{1}}\right) \beta_{1}\right)^{m n_{1} / \beta_{1}}+\left(1-\left(\frac{y_{i}-u_{2}}{\alpha_{2}}\right) \beta_{2}\right)^{m_{1} / \theta_{2}}+\left(1-\left(\frac{z_{i}-u_{3}}{\alpha_{3}}\right) \beta_{3}\right)^{2+1 / \beta_{3}}\right)^{1 / m_{11}-3} \\
& +\ln \left[\left(1-m_{\mathrm{i}}\right)\left(1-2 m_{\mathrm{t}}\right)+\left(\left(1-\left(\frac{x_{i}-u_{1}}{\alpha_{1}}\right) \beta_{1}\right) m^{m / \beta_{1}}+\left(1-\left(\frac{y_{i}-u_{2}}{\alpha_{2}}\right) \beta_{2}\right)^{m / \theta_{2}}\right.\right. \\
& \left.+\left(1-\left(\frac{z_{i}-u_{3}}{\alpha_{3}}\right) \beta_{3}\right)^{m_{1} / \beta_{3}}\right)^{2 / m_{1}}+\left(3 m_{1}-3\right)\left(\left(1-\left(\frac{x_{i}-u_{1}}{\alpha_{1}}\right) \beta_{1}\right)^{m_{1} / \beta_{1}}\right. \\
& \left.\left.+\left(1-\left(\frac{y_{1}-u_{2}}{\alpha_{2}}\right) \beta_{2}\right)^{m_{2} / \beta_{2}}+\left(1-\left(\frac{z_{i}-u_{3}}{\alpha_{3}}\right) \beta_{3}\right)^{m / \beta_{3}}\right)^{1 / m_{1}}\right]-\left(\left(1-\left(\frac{x_{i}-u_{i}}{\alpha_{1}}\right) \beta_{1}\right)^{m \nu / \beta_{1}}\right. \\
& \left.\left.\left.+\left(1-\left(\frac{y_{1}-u_{2}}{\alpha_{2}}\right) \beta_{2}\right){ }^{m / \beta_{2}}+\left(1-\left(\frac{z_{j}-u_{3}}{\alpha_{3}}\right) \beta_{3}\right)^{m_{1 / 1} / \beta_{3}}\right)^{1 / m_{1}}\right]\right\} \\
& +I_{4}\left(\sum _ { i = 1 } ^ { n _ { 4 } } \left[-\left(\ln \alpha_{\mathrm{r}}+\ln \alpha_{\mathrm{s}}\right)+\ln \left(1-\left(\frac{r_{i}-u_{\mathrm{r}}}{\alpha_{\mathrm{r}}}\right) \beta_{\mathrm{r}}\right)^{m_{\mathrm{b} 2} / \beta_{\mathrm{s}}-1}+\ln \left(1-\left(\frac{s_{i}-u_{3}}{\alpha_{s}}\right) \beta_{\mathrm{s}}\right)^{m_{\mathrm{m}} / \beta_{2}-1}\right.\right. \\
& +\ln \left(\left(1-\left(\frac{r_{i}-u_{\mathrm{r}}}{\alpha_{\mathrm{r}}}\right)_{\beta_{\mathrm{r}}}\right)^{m_{\mathrm{b} z} / \beta_{\mathrm{r}}}+\left(1-\left(\frac{s_{i}-u_{s}}{\alpha_{s}}\right) \beta_{s}\right)^{m_{1 \mathrm{~b} 2} / \beta}\right)^{1 / m_{\mathrm{b} 2}-7}+\ln \left(\left(m_{\mathrm{b} 2}-1\right)\right.
\end{aligned}
$$

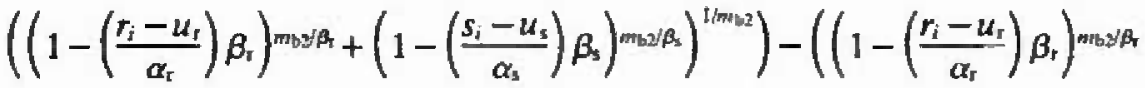

$$
\begin{aligned}
& \left.\left.\left.+\left(1-\left(\frac{s_{i}-u_{s}}{\alpha_{s}}\right) \beta_{3}\right) n^{n_{b} / \beta}\right)^{1 / m_{\mathrm{b} 2}}\right]\right\}+I_{5}\left\{-n_{5} \ln \alpha_{\mathrm{T}}\right. \\
& \left.+\sum_{i=1}^{n_{5}}\left[-\left(1-\left(\frac{r_{i}-u_{\mathrm{I}}}{\alpha_{\mathrm{i}}}\right) \beta_{\mathrm{t}}\right)^{1 / \beta_{\mathrm{H}}}+\ln \left(1-\left(\frac{r_{i}-u_{\mathrm{I}}}{\alpha_{\mathrm{r}}}\right) \beta_{\mathrm{r}}\right)^{1 / \beta_{\mathrm{T}}-1}\right]\right\}
\end{aligned}
$$

where:

$m_{\mathrm{t}} \quad$ trivariate association parameter

$m_{\mathrm{b} 1}, m_{\mathrm{b} 2}$ bivariate association parameter before and after the common period, respectively.

$\sum_{i=1}^{n_{1}} \ln f\left(p_{i}, \underline{\theta}_{1}\right)$ and $\sum_{i=1}^{n_{s}} \ln f\left(r_{i}, \underline{\theta}_{s}\right)$ take the form:

$$
\sum_{i=1}^{n_{j}}\left[-\ln \alpha_{\mathrm{t}}-\left(1-\left(\frac{t_{i}-u_{\mathrm{i}}}{\alpha_{\mathrm{i}}}\right) \beta_{i}\right)^{1 / \beta_{\mathrm{t}}}+\ln \left(1-\left(\frac{t_{i}-u_{1}}{\alpha_{\mathrm{t}}}\right) \beta_{i}\right)^{1 / \beta_{\mathrm{h}}-1}\right] .
$$


Similarly, $\sum_{i=1}^{n_{2}} \ln f\left(p_{i}, q_{i}, \underline{\theta}_{2}\right)$ and $\sum_{j=1}^{n_{4}} \ln f\left(r_{i}, s_{i}, \underline{\theta}_{4}\right)$ take the following form (bivariate relationship with both GEV marginals):

$$
\begin{aligned}
& \sum_{i=1}^{m_{t}}\left[-\left(\ln \alpha_{t}+\ln \alpha_{w}\right)+\ln \left(1-\left(\frac{t_{i}-u_{t}}{\alpha_{t}}\right) \beta_{l}\right)^{m_{t} / \beta_{t}-1}\right. \\
& +\ln \left(1-\left(\frac{w_{i}-u_{w}}{\alpha_{w}}\right) \beta_{w}\right)^{m+b_{w}^{\prime} \beta_{w-1}} \\
& +\ln \left(\left(1-\left(\frac{t_{i}-u_{1}}{\alpha_{t}}\right) \beta_{t}\right)^{m_{t} / \beta_{1}}\right. \\
& \left.+\left(1-\left(\frac{w_{i}-u_{w}}{\alpha_{w}}\right) \beta_{w}\right)\right)_{m i d Q_{w}}^{1 / m_{b}-2} \\
& +\ln \left(\left(m_{\mathrm{b}}-1\right)+\left(\left(1-\left(\frac{t_{i}-u_{i}}{\alpha_{\mathrm{t}}}\right) \beta_{\beta}\right) m^{m / \beta_{h}}\right.\right. \\
& \left.\left.+\left(1-\left(\frac{w_{i}-u_{w}}{\alpha_{w}}\right) \beta_{w}\right) m_{b} / \alpha_{w}\right)^{1 / m_{b}}\right) \\
& -\left(\left(1-\left(\frac{t_{j}-u_{\mathrm{T}}}{\alpha_{\mathrm{s}}}\right) \beta_{\mathrm{s}}\right)^{\mathrm{mb} / \beta_{\mathrm{k}}}\right. \\
& \left.\left.+\left(1-\left(\frac{w_{i}-u_{w}}{\alpha_{w}}\right) \beta_{w}\right)^{m_{w} / R_{w}}\right)^{1 / m_{b}}\right]
\end{aligned}
$$

Given the complexity of the mathematical expressions in Eq. (10) and their partial derivatives with respect to the parameters, the constrained multivariable Rosenbrock method, Kuester and Mize [10], was applied to obtain the maximum likelihood estimators for the parameters by the direct maximization of Eq. (10). The required initial values of the parameters to start the optimization of Eq. (10) were provided by the univariate maximum likelihood estimators of the parameters for the case of the location, scale, and shape parameters. The initial values of the association parameters, bivariate and trivariate, were set equal to 2, following the procedure developed by Escalante [8].

\section{Reliability of Estimated Parameters}

The indicator selected to measure the reliability of estimated parameters when using the trivariate distribution as compared with the univariate counterpart was the asymptotic relative information ratio.

\begin{tabular}{|c|c|c|c|c|c|}
\hline Parameter & $n_{4}$ & 0 & 25 & 50 & 75 \\
\hline \multirow{4}{*}{$w_{1}$} & 0 & 1.0868 & 1.3695 & 1.5055 & 1.5856 \\
\hline & 25 & 1.4460 & 1.5942 & 1.6823 & \\
\hline & 50 & 1.6295 & 1.7201 & & \\
\hline & 75 & 1.7408 & & & \\
\hline \multirow[t]{4}{*}{$a_{1}$} & 0 & 1.0141 & 1.2274 & 1.3256 & 1.3821 \\
\hline & 25 & 1.2712 & 1.3753 & 1.4356 & \\
\hline & 50 & 1.3941 & 1.4555 & & \\
\hline & 75 & 1.4662 & & & \\
\hline \multirow[t]{4}{*}{$\boldsymbol{\beta}_{1}$} & 0 & 1.2405 & 1.3555 & 1.4041 & 1.4312 \\
\hline & 25 & 1.3864 & 1.4382 & 1.4671 & \\
\hline & 50 & 1.4514 & 1.4806 & & \\
\hline & 75 & 1.4883 & & & \\
\hline \multirow[t]{4}{*}{$u_{z}$} & 0 & 1.0876 & 1.3722 & 1.5094 & 1.5903 \\
\hline & 25 & 1.0599 & 1,2263 & 1.3333 & \\
\hline & 50 & 1.0500 & 1.1683 & & \\
\hline & 75 & 1.0450 & & & \\
\hline \multirow[t]{4}{*}{$a_{2}$} & 0 & 1.0135 & 1.2151 & 1.3065 & 1.3587 \\
\hline & 25 & 0.9835 & 1.1046 & 1.1788 & \\
\hline & 50 & 0.9734 & 1.0604 & & \\
\hline & 75 & 0.9684 & & & \\
\hline \multirow[t]{4}{*}{$\beta_{2}$} & 0 & 1.2442 & 1.3469 & 1.3901 & 1.4140 \\
\hline & 25 & 1.1324 & 1.2069 & 1.2507 & \\
\hline & 50 & 1.0934 & 1.1507 & & \\
\hline & 75 & 1.0736 & & & \\
\hline \multirow[t]{4}{*}{$u_{3}$} & 0 & 1,0882 & 1.0462 & 1.0302 & 1.0217 \\
\hline & 25 & 1.0604 & 1.0417 & 1.0313 & \\
\hline & 50 & 1.0506 & 1.0390 & & \\
\hline & 75 & $\$ .0736$ & & & \\
\hline \multirow[t]{4}{*}{$a_{3}$} & 0 & 1.0126 & 0.9553 & 0.9334 & 0.9218 \\
\hline & 25 & 0.9822 & 0.9549 & 0.9395 & \\
\hline & 50 & 0.9790 & 0.9542 & & \\
\hline & 75 & 0.9669 & & & \\
\hline \multirow[t]{4}{*}{$\beta_{3}$} & 0 & 1.2437 & 1.0268 & 0.9535 & 0.9166 \\
\hline & 25 & 1.1314 & 1.0246 & 0.9704 & \\
\hline & 50 & 1.0923 & 1.0223 & & \\
\hline & 75 & 1.0724 & & & \\
\hline
\end{tabular}

Table 1 shows a sample of relative information ratios obtained by using the following set of parameters:

$$
\begin{array}{lll}
u_{1}=15.0, & \alpha_{1}=2.0, & \beta_{1}=-0.20 \\
u_{2}=12.0, & \alpha_{2}=1.2, & \beta_{2}=-0.15 \\
u_{3}=10.0, & \alpha_{3}=1.0, & \beta_{3}=-0.10
\end{array}
$$

Table 1. Asymptotic relative information ratios of the parameters of the TEV222 distribution for the maxima $n_{3}=25 ; m_{2}=2$; $m_{\mathrm{b} 2}=2$ 


\section{Case Study}

A region located in Northern Mexico, with a total of six gauging stations, was selected to apply the proposed methodology to the flood frequency analysis. Tables $2-4$ show the results of the application of the trivariate extreme value distributions for the maxima to the data recorded in such gauging stations.

In order to compare the goodness of fit between the univariate and trivariate maximum likelihood estimates of the parameters in stations considered in the case study, the standard error of fit, as defined by Kite [11], was obtained and is displayed in Table 5 .

Table 2. Correlation coefficients and relative sample sizes for the triplets of stations for the casc study

\begin{tabular}{lccccccc}
\hline \hline $\begin{array}{l}\text { Triplels of } \\
\text { stations }\end{array}$ & $\begin{array}{c}\text { Correlation } \\
\text { coefficicnt }\end{array}$ & \multicolumn{2}{c}{$\begin{array}{c}\text { Relative sample sizes } \\
n_{1}\end{array}$} & $n_{2}$ & $n_{3}$ & $n_{4}$ & $n_{5}$ \\
\hline Acatitan-Sta Cruz-1xpalino & 0.926 & 9 & 2 & 26 & 0 & 0 \\
Choix-Huitcs-Sn Francisco & 0.969 & 0 & 14 & 18 & 7 & 0
\end{tabular}

Table 3. Univariate maximum likelihood estimates of the parameters of the GEV distributions defined by the data of the gauging stations of the case study

\begin{tabular}{lrrr}
\hline \hline Station & Location & Scalc & Shape \\
\hline Acatitan & $\$ 76.21$ & 283.80 & -0.62 \\
Choix & 236.69 & 130.15 & -0.12 \\
Huitcs & 1564.78 & 978.87 & -0.57 \\
Lxpalino & 772.57 & 473.97 & -0.38 \\
Sn Francisco & 926.53 & 532.56 & -0.65 \\
Sta Cruz & 835.74 & 440.23 & -0.40 \\
\hline
\end{tabular}

Table 4. Trivariate maximum likelihood estimates of the parameters of the TEV222 distribution defined by the data of the gauging stalions of the case study

\begin{tabular}{lrrr}
\hline \hline Station & Location & Scalt & Shape \\
\hline Acatitan & 568.93 & 269.44 & -0.64 \\
Choix & 220.85 & 128.24 & -0.39 \\
Huites & 1603.30 & 1038.53 & -0.68 \\
Ixpalino & 795.03 & 490.86 & -0.46 \\
Sn Francisco & 943.69 & 540.73 & -0.67 \\
Sta Cruz & 850.97 & 467.74 & -0.52 \\
\hline
\end{tabular}

Table 5. Standard errors of fit for gauging stations of case study

\begin{tabular}{|c|c|c|}
\hline \multirow[b]{2}{*}{ Station } & \multicolumn{2}{|c|}{ Standard crror of fil } \\
\hline & $\begin{array}{c}\text { Univaritte } \\
\text { (GEV) }\end{array}$ & $\begin{array}{l}\text { Trivariate } \\
\text { (TEV222) }\end{array}$ \\
\hline Acatitan & $244.40^{\circ}$ & 253.90 \\
\hline Choix & 87.70 & $58.80^{\mathrm{a}}$ \\
\hline Huites & 1024.00 & $831.90^{\circ}$ \\
\hline Ixpulino & 537.90 & $393.00^{=}$ \\
\hline Sn Francisco & $350.80^{\mathrm{m}}$ & 401.50 \\
\hline Sta Cruz & 497.20 & $259.60^{y}$ \\
\hline
\end{tabular}

* Minimum standard crror of fit.

\section{Conclusions}

The logistic model for trivariate general extreme value distribution for the maxima has been proposed. Asymptotic and data base results suggest that the proposed model is a suitable option to be considered when performing flood frequency analysis.

\section{Acknowledgments}

The authors express their deepest gratitude to Comision Nacional del Agua, Universidad Nacional Autonoma de Mexico and Universidad Autonoma de Chihuahua for the support given for the publication of this paper. Funding for this study was provided by the Comision Nacional del Agua through the Agreement SARH-CNA-UNAM.

\section{References}

[1] B. V. Finkelstein, Limiting distributions of cxtreme tcrms of variational series of a two dimcissional random value, Dokl. Akad. Nauk. SSSR 91, 2 (1953).

[2] J. Tiago de Oliveira, Extremal disiributions, Faculdade de Ciencias de Lishna, 2 serie, A. Mat. VIl (1958).

[3] E. J. Gumbel, Distributions del valcurs extremes en plusiers dimensions, Publications de L'Institute de Statisli.que, Vol. 9, 17i-173 (1960).

[4] E. Rueda, Transfer of information for flood related variahter, M. Sc. thesis, Civil Enginecring Department, Culorado State University (1981) p. 112.

[5] J. A. Raynal, Bivariate extrerne valuc distributions applied Io flood frequency analysis, Ph. D. dissertation, Civil Engineering Department, CSU (1985) p. 237.

[6] J. Thago de Oliveira, Bivariate extremes: cxicnsions, Pruc. of the $40 \mathrm{th}$ session, Bulletin of the International StatisticaI Inslitute, Vol. 46, Book 2, 24l-25 I (1975).

[7] J. Tiagu de Olivcira, Bivariate and multivariate extreme Jistributions, Statistical Distributions in Scientific Work, G. P. Patil et al,, editors, D. Reidel Pubtishing Co., Vol. 1. (1975) pp. 355-361. 
[8] S. C. Escalante, Triva riate extreme value distributions and its applications to flood frequency analysis, $\mathrm{Ph}$. D. dissertation, Engineering Graduate Studies Division, Uniwersidad Nacional Autonoma de Mexico (1991) p. 315 (in Spanish).

[9] T. W. Anderson, Maximum likelihood estimates for a multivariate normal distribution when some observations are missing, Joumal of the American Statistical Association, Vol. 52, (1957) pp. 200-203.

[10] J. L. Kuester and J. H. Mize, Optimization with Fottran, McGraw Hill Book Co. (1973) pp. 386-398.

[11] G. W. Kite, Frequency and risk analysis in hydrology, Water Resources Publications (1977) p. 257.

About the authors: Carlos A. Escalante-Sandoval is an engineer at the Universidad Nacional Autonoma de Mexico and Jose A. Raynal-Villasenor is a mathematical statistician at the Universidad Autonoma de Chihuahua. 